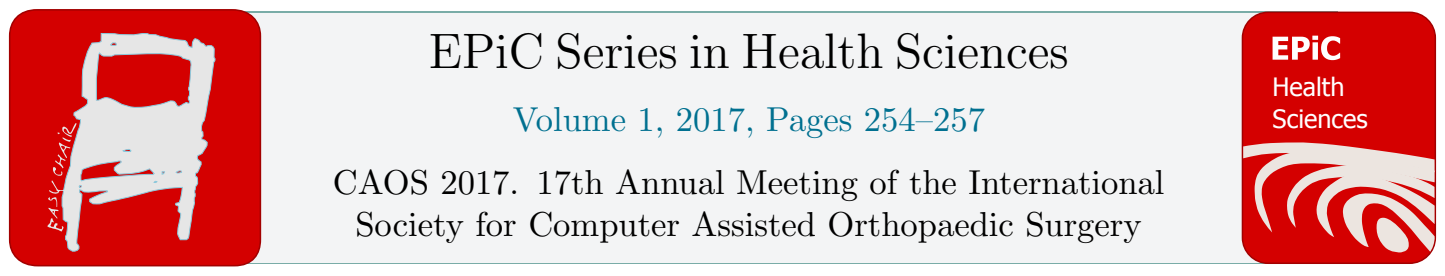

\title{
Integration of Robotic Applications in Open and Safe Medical Device IT Networks Using IEEE 11073 SDC
}

\author{
Manuel Vossel MSc ${ }^{1}$, Benjamin Strathen $\mathrm{MSc}^{1}$, Martin Kasparick Dipl.- \\ Inf. ${ }^{2}$, Meiko Müller Dipl.-Inf. ${ }^{1}$, Klaus Radermacher $\mathrm{PhD}^{1}$, Matías de la \\ Fuente $^{1}$, Armin Janß $\mathrm{PhD}^{1}$ \\ ${ }^{1}$ Chair of Medical Engineering, Helmholtz Institute for Biomedical Engineering, RWTH Aachen \\ University, Germany \\ ${ }^{2}$ Institute of Applied Microelectronics and Computer Engineering, University of Rostock, Germany \\ vossel@hia.rwth-aachen.de
}

\begin{abstract}
Modern operating rooms (OR) undergo a constant rise in the amount and complexity of technical systems. Due to a lack of inter-device communication and integration, each device works stand-alone resulting in redundant sensors, input devices, monitors and-last but not least—crowded ORs and error prone humanmachine-interaction. Therefore, various manufacturers such as Brainlab and Karl Storz provide proprietary integrated workstations. However, those "monolithic" solutions restrict the flexibility of the users and the clinical operators regarding integration of innovative third party devices.

In view of this, the OR.NET initiative (www.ornet.org) strives to develop international open standards for secure dynamic networks of medical devices in ORs. In the scope of the OR.NET project, based on service oriented architecture (SOA), the SDC (Service-oriented Device Connectivity) approach is currently in the process of standardization under IEEE 11073 to link medical devices in the OR (short OR.NETwork). It paves the way to interoperability between various medical devices due to its independence of license holders.

However, the SDC network does not suit real time (RT) requirements of a deterministic data transmission and low maximum latency, e.g. for robotic applications. This paper shows an approach to extend the secure dynamic OR by a real-time capable network to allow the integration of robotic systems. Exemplarily, this paper outlines an orthopaedic robotic system that is released by a universal configurable footswitch. This significantly extends the scope of applications for integrated ORs with the IEEE 11073 standard.
\end{abstract}




\section{Introduction}

Modern operating rooms (OR) undergo a constant rise in the amount and complexity of technical systems. Due to a lack of inter-device communication and integration, each device works stand-alone resulting in redundant sensors, input devices, monitors and-last but not least-crowded ORs and error prone human-machine-interaction. Therefore, various manufacturers such as Brainlab and Karl Storz provide proprietary integrated workstations (Ibach 2006). However, those "monolithic" solutions restrict the flexibility of the users and the clinical operators regarding the integration of innovative third party devices. In view of this, the OR.NET initiative (www.ornet.org) strives to develop international open standards for secure dynamic networks of medical devices in ORs (Kasparick 2015).

In the scope of the OR.NET project, based on service oriented architecture (SOA), the SDC (Service-oriented Device Connectivity) approach is currently in the process of standardization under IEEE 11073 to link medical devices in the OR (short OR.NETwork). It paves the way to interoperability between various medical devices due to its independence of license holders. This highly flexible network standard incorporates plug-and-play, scalability, interoperability, reusability, and represents an innovative basis for safe, secure and open networks of modular medical devices in the operating room.

However, the SDC network does not suit real time (RT) requirements of a deterministic data transmission and low maximum latency, e.g. for robotic applications. Whereas this may also hold for other devices such as powertools or footswitches, RT requirements for robot motion control using external (OR.NETwork based) position data certainly induces most severe constraints. In a SOA based integrated modular network supporting data exchange between service providers and service consumers, e.g. a central integrated tracking system could provide dynamic position data as a service provider to different systems and applications (service consumers) in the OR. Whereas a central tracking server based on non-RT web services could be sufficient for registration or even freehand navigation apps (Ibach 2009), this would not be an adequate solution for RT robotic motion control (Benzko 2010). Therefore, we followed the approach to use EtherCAT, nowadays already offered as a standard port by many commercial motion controllers. This contribution proposes the approach to use the latest generation of high-speed optical tracking systems such as the fusionTrack 500 (Atracsys LLC, Puidoux, $\mathrm{CH}$ ) as a central integrated tracking device in an OR.NETwork and EtherCAT based RT communication for robot motion control.

\section{Proposed Approach}

The main objective of our work is to integrate the modular mini-robot for minimal invasive orthopaedic surgery (MINARO), developed at the Chair of Medical Engineering at the RWTH Aachen University (Niggemeyer 2012). Modular mechanics and control architecture of the MINARO system enable an adaptation of the workspace and performance of the modular robot system to the application specific needs. Depending on the application scenario, even the medical staff could configure the autoclavable robotic system prior to the intervention. Due to the OR.NETwork, the surgical central workstation can be used as human-computer-interface to display and interact with related planning, registration and navigation information of the MINARO system and it can provide all relevant interfaces for retrieval and storage of images and documentation to the clinical PACS and HIS systems.

For a closed loop robot control, input from various sensors like high-speed optical tracking, inertial sensors, and robot axis encoders have to be processed and matched with the target robot position as well as the planned trajectory. Sensor data must be transmitted and processed with a high 
temporal resolution and low network latencies and jitter. Based on those requirements, the architecture as presented in Figure 1 was designed. A connector is under development to connect the real-time EtherCAT network with non-real-time SDC networks in the overall OR.NET architecture.

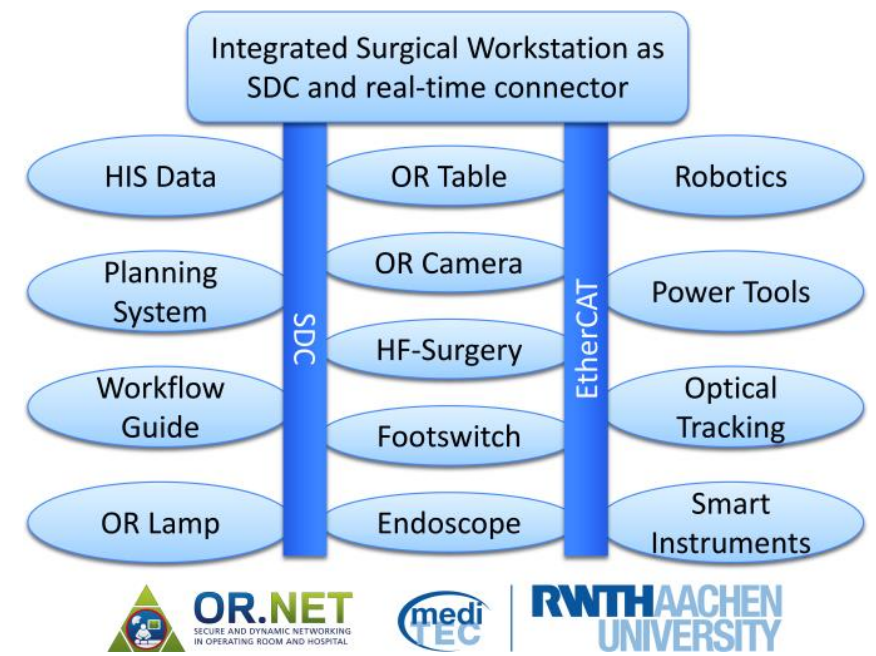

Figure 1: Initial (parallel EtherCAT network) of an integrated surgical workstation and OR.NETwork incorporating RT capabilities.

Some devices, such as footswitches, are connected to both networks, the real-time EtherCAT as well as the non-real-time SDC. E.g., in case of a robotic removal of the femoral bone cement in revision total hip replacement (Niggemeyer 2012), real-time capability of the footswitch is used to enable the surgeon to stop and restart the robotic milling process anytime. In contrast, the SDC connection is used e.g. to change the configuration of the programmable footswitch depending on the sequence of intervention (e.g., the same footswitch may be used for the interaction with the navigation system - e.g., for registration-, the C-Arm or US imaging etc.). The configuration can be performed on the surgical workstation as seen in Figure 2, based on previous works from Dell'Anna 2016.

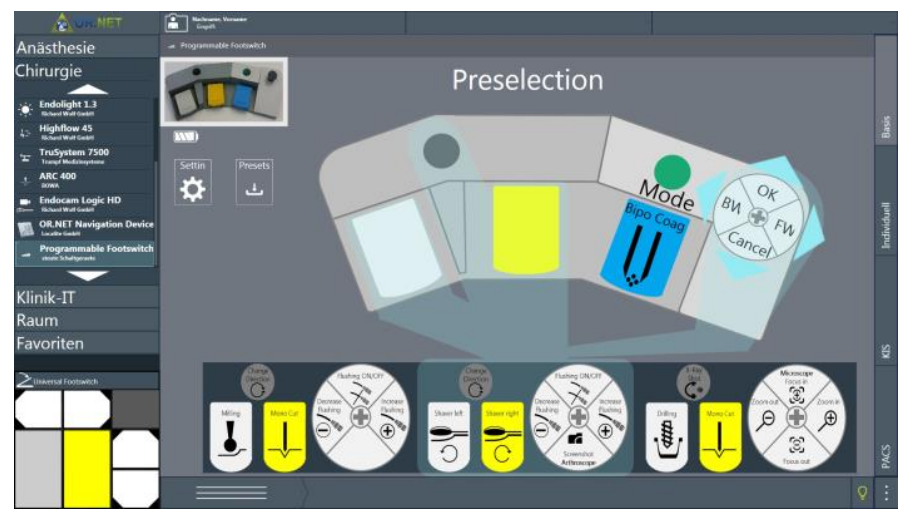

Figure 2: Configuration of the universal footswitch on the Surgical Workstation. 


\section{Outlook}

As shown in this paper, the safe, secure and dynamic OR.NETwork can be extended by an RT network to allow the integration of robotic systems using central services, e.g. from an integrated high-speed tracking system or a universal footswitch unit. This significantly extends the scope of applications for integrated ORs with the proposed IEEE 11073 standards (Benzko 2010). However, this approach introduces a second, parallel network which increases the amount of cables in the OR and accordingly complexity. Therefore, the same physical layer shall be used for the present SDC network and real-time traffic (RT-SDC in Figure 1) in its final implementation (Cucinotta 2009). The evaluation of this approach and the related extension of the IEEE 11073 standard family to incorporate RT capability are major aspects of our ongoing work.

\section{References}

Benzko J, Ibach B, Niggemeyer M, Radermacher K, A novel SOA based approach towards the integration of a robotic system into a modular surgical work system and IT network, International Journal of Computer Assisted Radiology and Surgery, 5(Supplement 1), pp.193-194, 2010.

Cucinotta T, Mancina A, Anastasi GF, Lipari G, Mangeruca L, Checcozzo R, Rusinà F, A realtime service-oriented architecture for industrial automation, IEEE Transactions on Industrial Informatics, 5(3), pp.267-277, 2009.

Dell'Anna J, Janß A, Clusmann H, Radermacher K, A Configurable Footswitch Unit for the Open Networked Neurosurgical OR - Development, Evaluation and Future Perspectives, I-Com, 15(3), pp.227-247, 2016.

Ibach B, Benzko J, Janß A, Radermacher K, Sharing tracking data between different systems using an Open Surgical Communication Bus, In O. Dössel, W. C. Schlegel, eds. World Congress on Medical Physics and Biomedical Engineering, September 7 - 12, 2009, Munich, Germany. Springer, pp. 21-23. 2009.

Ibach B, Zimolong A, Portheine F, Niethard FU, Radermacher K, Integrated medical workstations for Computer Integrated Smart Surgery (CISS) - State of the art, bottlenecks and approaches, International Journal of Computer Assisted Radiology and Surgery, 1(SUPPL. 7), pp.449-451, 2006.

Kasparick M, Schlichting S, Golatowski F, Timmermann D, Medical DPWS: New IEEE 11073 standard for safe and interoperable medical device communication, In 2015 IEEE Conference on Standards for Communications and Networking (CSCN). pp. 212-217. 2015.

Niggemeyer M, Müller M, Niesche A, De La Fuente M, Komadinic A, Radermacher K, Modular design of a miniaturized surgical robot system, Biomedizinische Technik, 57(4), pp.261-268, 2012.

\section{Acknowledgements}

This research has been funded in parts within the projects OR.NET - Secure and Dynamic Networking in Operating Room and Hospital (German Federal Ministry of Education and Research BMBF; grant no.: 16KT1203 and 16KT1238) and the project ZiMT - Certifiable Integrated Medical Technology and IT Systems based on Open Standards in the Operating Room and Hospital (State of North-Rhine Westphalia and the European Commission - European Regional Development Fund (EFRE); grant no.: EFRE-0800454). 